\title{
Sudden onset of the leg swelling after endoscopic knee surgery
}

\author{
Yoshio Misawa $^{1 *}$ and Koji Kawahito ${ }^{2}$
}

Misawa Y, Kawahito K. Sudden onset of the leg swelling after endoscopic knee surgery. J Phlebol Lymphol. 2018;11(1):34-38.

Minimally invasive surgery has been increased worldwide. We experienced with a case of an iatrogenic popliteal arteriovenous fistula after endoscopic knee surgery.

A 70-yeay old ma underwent endoscopic resection of a Baker cyst in the left knee joint. The left leg swelling suddenly occurred seven days after the surgery. Computed tomographic study of the legs confirmed the diagnosis, and he successfully underwent repair surgery of the fistula and aneurysm.

\section{INTRODUCTION}

Minimally invasive surgery, including endoscopic knee surgery, has been increasing worldwide. The approach is associated with less postoperative rehabilitation and early return to activities of daily living. However, complications occur, some immediately and others long after the surgery. We experienced a patient with iatrogenic popliteal arteriovenous fistula after endoscopic knee surgery, which occurred 1 week postoperatively.

\section{CASE}

A 70-year old man underwent endoscopic resection of a Baker cyst in the left knee joint. Postoperative rehabilitation began the day after the operation, with the patient able to stand and walk by himself. Seven days after the surgery, he presented with sudden swelling and pain in his left leg. Because of the sudden onset of the clinical signs, we considered vascular complications. Computed tomographic imaging of the lower extremities revealed swelling in the left leg and fistula between the left popliteal artery and vein with a popliteal artery aneurysm (Figures 1A and 2B). The patient's complications were successfully resolved by closing the fistula and resection of the aneurysm. His preoperative symptoms and signs completely subsided 1 week after the fistula and aneurysm repair. He returned to his ordinary daily life 2 weeks after the surgery, and no other complications were detected during 6 months thereafter (Figures $1 \mathrm{C}$ and 1D).

\section{DISCUSSION}

Minimally invasive surgery, including endoscopic knee surgery, has been increasing worldwide and is associated with less postoperative rehabilitation. Generally, mobilization and/or exercise of the extremities could lead to swelling of them after periods of rest. Progress in surgical techniques has changed rehabilitation medicine and postoperative care for orthopedic patients, and postoperative rehabilitation now tends to begin earlier postoperatively.

Iatrogenic vascular injuries could include hemorrhage, aneurysmal formation, and arteriovenous fistula. Also, injury-related masses may cause compression effects in adjacent organs such as nerves and muscles, and arteriovenous drainage has a possible risk of limb congestion with or without congestive heart failure.

${ }^{1}$ Department of Rehabilitation, Utsunomiya Rehabilitation Hospital, Utsunomiya, Tochigi, Japan

${ }^{2}$ Division of Cardiovascular Surgery, Department of Surgery, Jichi Medical University, Shimotsuke Tochigi, Japan E-mail: misawa.y@utsunomiyarhhp.jp ; Doi: 10.14303/1983-8905.1000056

Received: July 19, 2018, Accepted: July 27, 2018, Published: August 03, 2018
Iatrogenic vascular injuries can include hemorrhage, aneurysmal formation, and arteriovenous fistula. Also, injury-related masses may cause compression in adjacent organs such as nerves and muscles, and arteriovenous drainage has a possible risk of limb congestion with or without congestive heart failure. This rare complication of an arteriovenous fistula should be considered when sudden leg swelling is encountered after surgery.

Key Words: Popliteal artery aneurysm, Popliteal arterio-venous fistula, Endoscopic knee surgery, Acute leg swelling.

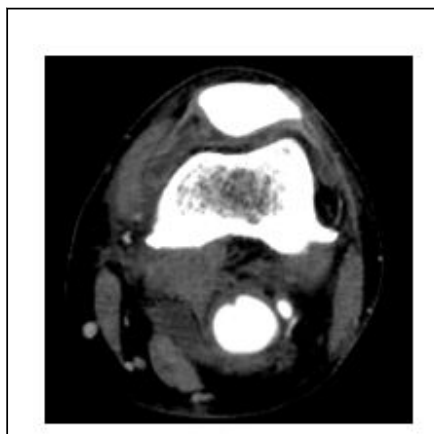

$1 \mathrm{~A}$

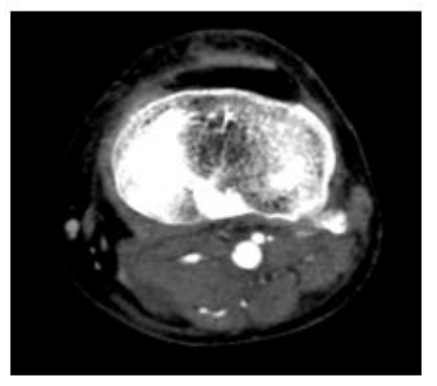

$1 \mathrm{C}$

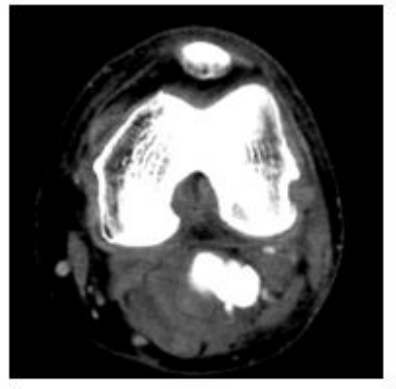

1B

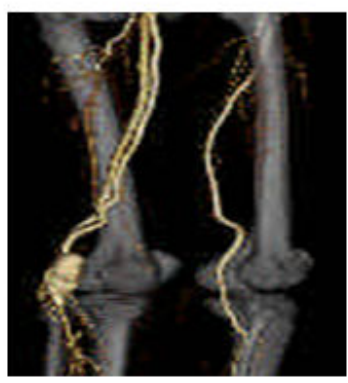

1D
Figure 1: Preoperative computed tomographic findings of the legs

Surgery-related clinical manifestations can occur at different periods after knee surgery. Our case developed complications 7 days after this surgery; however, a previously reported patient developed complication 3 years after total knee arthroplasty and the patient underwent successful open surgical repair. Bowman et al. [1] reported popliteal arterial pseudoaneurysm and arteriovenous fistula after tibial lengthening osteotomy in a 7-year-old boy without an exact latent period.

As a treatment method, Burger et al. [2] described percutaneous endovascular treatment of iatrogenic arteriovenous fistula in two different patients, one with a popliteal lesion and another with an iliac lesion. The \footnotetext{
This open-access article is distributed under the terms of the Creative Commons Attribution Non-Commercial License (CC BY-NC) (http://
creativecommons.org/licenses/by-nc/4.0/), which permits reuse, distribution and reproduction of the article, provided that the original work is properly cited and the reuse is restricted to noncommercial purposes. For commercial reuse, contact reprints@pulsus.com
} 
authors successfully performed repairs with simultaneous intravascular ultrasound. Kane et al. [3] also experienced a fistula patient following stent placement during interventional radiology.

From 17 cases with peripheral vascular lesions, Onal et al. [4] concluded that endovascular treatment of various types of peripheral vascular lesions with stent-grafts was a low-risk procedure.

The study consisted of 10 cardiac catheterization-induced femoral arteriovenous femoral fistulas, an iatrogenic iliac fistula, one penetrating trauma-induced femoral fistula, two penetrating trauma-induced pseudoaneurysms of the axillary and popliteal arteries, and three nontraumatic iliac arterial aneurysms. All 17 patients successfully underwent endovascular surgery. Less invasive treatments for these complications are preferable, but open surgical repair is also adequate for select complicated patients such as ours [5].

\section{CONCLUSION}

A 70-year-old man presented with symptoms of swelling and pain in the operated leg 7 days after endoscopic knee surgery. Computed tomographic imaging confirmed a fistula between the popliteal artery and vein, with a popliteal arterial aneurysm. The patient underwent successful open surgical repair for the complications. This rare complication should be considered when sudden leg swelling is encountered during postoperative rehabilitation.

\section{ACKNOWLEDGMENT}

We thank Jane Charbonneau, DVM, from Edanz Group (www.edanzediting.com/ac) for editing a draft of this manuscript.

\section{CONFLICTS OF INTEREST}

We disclose neither commercial affiliation nor consultancy that could be construed as a conflict of interest with respect to the submitted data.

\section{REFERENCES}

1. Bowman JN, Ellozy SH, Ting J, et al. Successful repair of popliteal artery pseudoaneurysm after tibial lengthening osteotomy in a 7 year-old boy. Vasc Endovascular Surg. 2008;42(6):610-14.

2. Burger T, Meyer F, Tautenhahn J, et al. Percutaneous treatment of rare iatrogenic arteriovenous fistulas of the lower limbs. Int Surg. 1998;83(3):198-01.

3. Kane I, Post Z, Ong A, et al. Arteriovenous fistula formation after intra-articular injection following total joint arthroplasty. Orthopedics. 2016;39(5):e976-e79.

4. Onal B, LLgit ET, Koşar S, et al. Endovascular treatment of peripheral vascular lesions with stent-grafts. Diagn Interv Radiol. 2005;11(3):170-74.

5. Thomas R, Agarwal M, Lovell M, et al. An unusual presentation of a popliteal arteriovenous fistula after primary total knee arthroplasty. J Arthroplasty. 2008;23(6):945-48. 